\title{
THEORY OF WOLF-RAYET ATMOSPHERES
}

\author{
D. John Hillier \\ Joint Institute for Laboratory Astrophysics \\ University of Colorado and National Institute of Standards and Technology \\ Boulder, Colorado 80309-0440 \\ USA
}

\begin{abstract}
Theoretical modeling of line and continuum formation in WolfRayet (W-R) atmospheres is reviewed. We examine the basic premises on which the models are built, and critically compare theoretical models with observation. Discrepancies between theory and observation are analyzed to determine their implication for our understanding of W-R atmospheres. Line formation, continuum formation, and the ionization structure of W-R envelopes are examined in detail. Abundance determinations are briefly reviewed.
\end{abstract}

\section{INTRODUCTION}

The idea that W-R stars consist of a hot core surrounded by a dense stellar wind has been in existence for many years (e.g. Beals 1944, Rublev 1964) but only recently have techniques been developed to allow this theory to be examined in detail. With the assumptions of spherical geometry and homogeneity, it is now possible to self consistently solve the transfer problem in moving atmospheres, allowing us to make a detailed quantitative comparison of theoretical models with observation.

Below we examine recent theoretical modeling of $\mathrm{W}-\mathrm{R}$ atmospheres. In $\S 2$ we describe the Standard Model, and examine the basic premises on which it is built. Pure helium models of W-R stars are introduced in $\$ 3$. This lays the foundation for more detailed discussion of continuum formation ( $(4)$, and line formation ( $\$ 5)$. The ionization structure is examined in $\S 6$, with particular regard to comparison of theory with observation. In $\$ 7$ we present a critical discussion regarding the current status of the Standard Model - we describe its successes and its failures. The current status of abundances in W-R stars is briefly reviewed in $\S 8$, while in $\S 9$ we critically examine the accuracy of the stellar parameters.

\section{STANDARD MODEL}

The models to be examined are primarily those of Hillier $(1983,1987 \mathrm{a}, \mathrm{b}, 1988,1989)$ and the Kiel group - Hamann, Schmutz, and Wessolowski - in Germany (Hamann 1985, Schmutz and Hamann 1986, Hamann and Schmutz 1987, Wessolowski, 
Schmutz, and Hamann 1988, Hamann, Schmutz, and Wessolwoski 1988, and Schmutz, Hamann, and Wessolowski 1989). In these models the observed W-R emission line spectrum arises in a dense stellar wind which is photoionized by a hot core. The Standard Model assumes

(i) spherical geometry,

(ii) a monotonic velocity law,

(iii) homogeneity,

(iv) and time independence (stationary).

In the models of Hillier $(1983,1987 \mathrm{a}, \mathrm{b})$, radiative equilibrium was assumed, although the temperature in the outer regions of the stellar wind could be adjusted to allow for cooling by metal lines, or mechanical energy deposition. The models by the Kiel group assume a gray temperature distribution (slightly modified at large radii) (e.g., Wessolowski, Schmutz, and Hamann 1988) which is in rough agreement with the radiative equilibrium temperature found in more sophisticated calculations. Both groups can now compute full radiative equilibrium models which include cooling due to N, C and O (e.g., Hillier 1989). In the models to date, line blanketing has been neglected.

The Standard Model is probably the simplest model that can be offered to explain the spectral appearance of W-R stars. Its assumptions are well defined and importantly, the radiation transfer equation and the statistical equilibrium equations are solved self consistently. These models provide important insights into line and continuum formation processes in W-R atmospheres - insights which can only be gained from detailed transfer calculations. Only by generating such models can we hope to understand W-R atmospheres: They are a prerequisite to all future modeling.

\section{THE He I/He II MODELS OF WOLF-RAYET STARS}

The first detailed model of a W-R star was that of Hillier $(1983,1987 \mathrm{a}, \mathrm{b})$, who modeled the WN5 star HD 50896. He found that the continuous energy distribution, the He I spectrum, and the He II spectrum could be matched by a model with $R_{*}=$ $2.5 R_{\odot}, \dot{M}=5.0 \times 10^{-5} M_{\odot} \mathrm{yr}^{-1}$ and an effective temperature of $55000 \mathrm{~K}(L=$ $\left.5 \times 10^{4} L_{\odot}\right)$ to $65000 K\left(L=10^{5} L_{\odot}\right)$. The distance was a free parameter (the above model corresponds to $1.2 \mathrm{kpc}$ ) and could not be derived spectroscopically - models with different luminosities, mass loss rates and core radii yielded similar spectra (see below).

The relative strengths of the $\mathrm{He} \mathrm{I}$ and $\mathrm{He}$ II lines are primarily determined by the ionization structure and it is the ratio of these two strengths that allows us to determine the effective temperature of the underlying star. Stars that show appreciable emission in He I $\lambda 5876$ in their spectra will have $\mathrm{He}^{+}$as the dominant ionization state of helium in the radio region (Hillier 1983,1987a, Schmutz and Hamann 1986). The presence of He I thus provides an upper limit to the effective temperature of the underlying star (Schmutz and Hamann 1986).

To match the He I line strengths in HD 50896 the low luminosity model was preferred, however with the inclusion of metal cooling (Hillier 1989) the high luminosity 
model is now preferred. Cooling due to lines of $\mathrm{N}$ and $\mathrm{C}$ decreases the electron temperature in the He I formation region from the $30000 \mathrm{~K}$ adopted by Hillier (1987b) to approximately $15000 \mathrm{~K}$ (Hillier 1989) which increases the strength of the He I lines. (The He I lines are formed by recombination, and thus their strength scales roughly as $1 / \mathrm{T}$.)

Hamann, Schmutz, and Wessolowski (1988) have also modeled the spectrum of HD 50896 in detail. They considered three models corresponding to different adopted distances between 0.7 and $2 \mathrm{kpc}$ - all models have a similar effective temperature of approximately $60000 \mathrm{~K}$. Their model B is similar to that of Hillier, and they reach essentially the same conclusions regarding the mass loss rate, luminosity, and core radius. Based on analysis of the interstellar $\mathrm{NaD}$ line, a distance of $>1.6 \mathrm{kpc}$, corresponding to Hamann, Schmutz, and Wessolowski's model A, is to be preferred (Schmutz and Howarth, these proceedings).

Hamann and Schmutz (1987) computed a systematic grid of He II spectra for W-R stars, illustrating the variation of line profiles and line strengths with various parameters, e.g., $\dot{M}, T_{\text {eff }}, R_{*}$. A similar analysis for He I was performed by Wessolowski, Schmutz, and Hamann (1988).

From their detailed numerical calculations Schmutz, Hamann, and Wessolowski (1989) found that approximately the same line equivalent widths could be produced in different models provided that $\dot{M}, V_{\infty}$, and $R_{*}$ were related. They used a transformed radius $R_{t}$ to relate these parameters:

$$
R_{t}=R_{*}\left(\frac{V_{\infty}}{2500 \mathrm{~km} \mathrm{~s}^{-1}}\right)^{2 / 3}\left(\frac{10^{-4} M_{\odot} \mathrm{yr}^{-1}}{\dot{M}}\right)^{2 / 3}
$$

Properties of individual stars could thus be read from a single grid of $\mathbf{R}_{t}$ versus $T_{\text {eff }}$. To derive the stellar parameters of WN stars $\left(T_{\text {eff }}, \dot{M}, R_{*}\right)$ four scaler parameters are required - the absolute flux in one continuum band, the equivalent width of one He II line, the equivalent width of one He I line, and the terminal velocity (as indicated by the He I and He II line profiles) of the stellar wind.

The inclusion of $\mathrm{N}$ and $\mathrm{C}$ in WN stellar models has only a small effect on the derived parameters of WN stars (Hillier 1989, Hamann and Wessolowski 1990) the inclusion of these species primarily affects the energy balance and hence the equilibrium wind temperature.

On the other hand, inclusion of $\mathrm{C}$ in WC star models has a significant effect. This is not surprising as the $\mathrm{C} / \mathrm{He}$ abundance (by number) in WC stars is greater than 0.1 (Nugis 1982b, Torres 1988, Smith and Hummer 1988, Hillier 1989). Schmutz, Hamann, and Wessolowski (1989) derived an effective temperature of $35000 \mathrm{~K}$ for HD 165763 (WC5). With the inclusion of carbon, Hillier (1989) found that a model with an effective temperature of $60000 K$ gave the best agreement with observations.

\section{CONTINUUM FORMATION}

Hillier $(1983,1987 \mathrm{a})$ has made a detailed investigation into formation of the continuum in HD 50896. These models emphasize the insensitivity of the continuum to effective temperature $\left(T_{e f f}\right)$, and the importance of other processes in determining 
the observed continuum shape. We cannot derive effective temperatures from the continuous energy distribution - a comparison of the model energy distribution with that observed can only rule out models, it cannot validate them. The insensitivity of the continuum to the effective temperature for $\mathrm{O}$ stars has been discussed by Abbott and Hummer (1985).

We define $T_{\text {eff }}$ relative to the stellar radius where the velocity has become subsonic. This may be considerably different from the classic definition in which the radius is chosen at Rosseland optical depth $2 / 3$. This radius can occur well out in the stellar wind, and the temperature so defined in no way reflects the quality of the radiation field - the $T_{\text {eff }}$ defined at $R_{*}$ is much better. Note that $T_{e f f}$ is merely a parameterization - it does not mean that the emitted spectrum can be approximated by a blackbody of this temperature. W-R spectra clearly show excesses in the visual and IR, and most do not emit any flux shortward of the He II Lyman limit.

At low mass loss rates the visual flux is proportional to $R_{*}^{2}$ and $T_{\text {eff }}$ (as on Rayleigh-Jeans tail of blackbody), but as the mass loss rate increases the visual magnitude becomes increasingly influenced by the stellar wind (see Hillier 1987a). For the hot W-R stars (e.g. WN2-WN6, WC4-WC7) the bulk of the flux is emitted shortward of $912 \AA$ - in the $10^{5} L_{\odot}$ model of HD $50896,75 \%$ of the stellar flux is emitted below the Lyman limit, and hence is unobservable. Schmutz, Hamann, and Wessolowski (1989) provide a detailed grid of models illustrating the variation of absolute visual magnitude as a function of $\dot{M}, R_{*}$, and $T_{\text {eff }}$.

The agreement with observation (see Schmutz, this symposium) is excellent, especially when allowance is made for the neglect of line blanketing and observational error (particularly the correction due to reddening). The radio and IR fluxes are also predicted by these models (e.g., Hillier 1987b) - there is excellent agreement with the IR fluxes $(\lambda \leq 10 \mu \mathrm{m})$ while the radio fluxes may be systematically overestimated by up to a factor of 2 (Hamann, Schmutz and Wessolowski 1988, Hillier 1989). For HD 50896 (with $\mathrm{d}=1.2 \mathrm{kpc}$ ) Hillier (1987b) derived a mass loss rate of $5.0 \times 10^{-5} M_{\odot} \mathrm{yr}^{-1}$, whereas from the radio data of Hogg (1989) a mass loss rate of $3.5 \times 10^{-5} M_{\odot} \mathrm{yr}^{-1}$ is derived. The reason for the discrepancies is unknown better agreement is obtained if we adopt the larger terminal velocities derived from UV P Cygni profiles rather than those obtained from fitting He I profiles. This problem needs to be re-examined using detailed spectral analysis with the more advanced model calculations now available.

There is a suggestion in some recent observations by Hogg (1989) of some peculiar spectral indices in the radio region (considering only those stars believed to have thermal winds) but as these results are only $2 \sigma$, higher signal-to-noise observations are required. It is very important that high signal-to-noise IR and radio flux measurements, such as the $1100 \mu \mathrm{m}$ IR flux for the WC8 star $\gamma$ Vel recently measured by Williams et al. (1990), be obtained in order to provide checks on the modeling. Such measurements can place important constraints on the ionization structure, the velocity law, and inhomogeneities in the stellar wind. Likewise, important constraints can be found from radio observations which resolve the stellar wind (e.g., Hogg 1985). 


\section{LINE FORMATION, AND THE STRATIFICATION OF THE LINE EMISSION WITHIN THE EXTENDED W-R ATMOSPHERE}

Although we are discussing line and continuum formation separately I wish to stress that these processes are not independent, and must be modeled simultaneously to match observations.

The Standard Model is capable of matching both the line strengths and emission line profiles seen in W-R spectra. Model fits of line profiles with observation are given by Hillier $(1987 a, 1988)$ and Hamann, Schmutz and Wessolowski (1988), while a comprehensive comparison of predicted line equivalent widths is given by Hillier (1987b,1989). Both the Gaussian He II profiles, and the flat topped He I profiles are reproduced by the Standard Model.

Neither Hillier nor the Kiel group has attempted to fine tune the models to systematically fit the observed profiles - even if such a procedure could be done it is unwarranted because of limitations in the models (e.g., the neglect of line blanketing), because of line variability, because of line blending and observational error, and because fine tuning would not affect the results of the analyses. In $\S 7$ we examine those differences between theoretical and observed profiles which we believe to be of significance for our understanding of W-R stellar winds.

Using the Sobolev approximation, it is possible to illustrate the regions from which various emission lines arise (e.g., Hillier $1987 \mathrm{~b}, 1988,1989)$. This is very useful since it allows one to determine the local properties affecting the strengths of particular emission lines. In a given series (i.e., n-4 Pickering series), the optical depth decreases with upper principal quantum number, and consequently these lines are formed deeper within the stellar envelope (see Hillier, Jones, and Hyland 1983).

The principal processes leading to $\mathrm{W}-\mathrm{R}$ emission lines are:

(i) radiative recombination,

(ii) collisional excitation,

(iii) continuum fluorescence and

(iv) dielectronic recombination.

These processes, together with examples of lines formed by each mechanism, are discussed in detail by Hillier (1990a). By understanding line formation processes we are in a better position to determine those lines best suited for abundance determinations, and those lines best suited to provide diagnostics of the atmospheric structure.

The variable ionization structure $(\S 6)$ has important implications for line formation in the stellar wind. Lines from different ions generally arise in different regions of the stellar wind, and hence their strengths are influenced by different physical conditions. In early W-R stars the He I recombination lines arise at larger radii, and at lower electron temperatures than He II recombination lines. As the recombination rates have an approximate inverse dependence on $T_{e}$, the He I emission lines are enhanced relative to He II. In addition, in early W-R stars the He I lines tend to be optically thin, whereas the strengths of the He II lines are influenced by optical depth effects. Similarly, in HD 165763 (WC5), Hillier (1989) found that the C II and C III recombination lines originate at larger radii than the C IV recombination lines. 


\section{IONIZATION STRUCTURE}

In the models of Schmutz and Hamann (1986), Hamann and Schmutz (1987), Schmutz, Hamann, and Wessolowski $(1989)$ and Hillier $(1987 a, b, 1988,1989)$ the ionization of the envelope decreases outward. This is a firm prediction of the models, and provides a simple explanation for the flat-topped profiles of $\mathrm{He}$ I and C III, the observed $10 \mu \mathrm{m}-6 \mathrm{~cm}$ spectral index, and the observed line strengths.

The models predict a correlation of ionization potential with line width (Hillier 1988,1989), as observed (Kuhi 1973). This is in agreement with the standard picture that the ionization in a W-R envelope decreases outward (Beals 1944, Kuhi 1968). Note this is not an indication of the wind temperature - rather the outward decreasing ionization state of the envelope arises from dilution of the continuum radiation and the important role of photoionizations from excited states.

Ionizations from excited states are of critical importance in determining the ionization of the envelope - arguments by Antokhin, Kholtygin, and Cherepaschuk (1988) that the observed $\mathrm{He}$ I and He II line strengths require all W-R stars to have similar effective temperatures are invalid. This is convincingly demonstrated above, and in the paper by Schmutz, Hamann, and Wessolowski (1989) where a spectral analysis of $30 \mathrm{~W}-\mathrm{R}$ stars is presented. Likewise the observed ionization of other species in W-R spectra does not, at this time, provide convincing evidence that an alternative model of the emission line region is required.

Close to the surface of a WN5 or WC5 star, $\mathrm{He}^{2+}, \mathrm{C}^{4+}, \mathrm{N}^{5+}$ and $\mathrm{O}^{6+}$ are the dominant ionization stages. As we move further out in the envelope the ionization systematically decreases so that in a WN5 star, $\mathrm{He}^{+}, \mathrm{C}^{3+}, \mathrm{N}^{3+}$, and $\mathrm{O}^{3+}\left(\right.$ or $\mathrm{O}^{2+}$ ) eventually become the dominant ionization stages. In HD 165763 (WC5), $\mathrm{He}^{+}$and $\mathrm{C}^{2+}$ are likely to be the dominant ionization stages in the radio region. The large optical depth in the He II Lyman continuum (i.e., $\lambda<228 \AA$ ), the large optical depths in the resonance lines coupled with the extreme UV continuous radiation field, and ionizations from excited states play a fundamental role in determining the ionization structure of the envelope. The predicted ionization structure is illustrated by Hillier (1988) for WN stars, and by Hillier (1989) for WC stars.

The inclusion of metals in the H/He W-R models of Hillier, and the Kiel group is extremely significant as it provides an important consistency check of the models. Discrepancies in model N/C/O line strengths (as a function of ionization state) will indicate deficiencies of the stellar model.

The present calculations are unable to simultaneously match all stages of ionization - in particular in the WC model of HD 165763 the O VI doublet at $\lambda \lambda 3811,3834$ is too weak, indicating that a higher ionization model is required. A model with twice the luminosity matches better the $\mathrm{O}$ VI lines (but is still too weak) but then underestimates the strength of the He I lines by a factor of 2 . Similarly, a model that fits the $\mathrm{He}$ I/He II ratio in HD 50896 underestimates the strength of the N V $\lambda \lambda 4604,4620$ doublet (Hillier 1988). The inclusion of line blanketing may resolve these discrepancies. 


\section{STANDARD MODEL - SUCCESSES AND FAILURES}

The Standard Model is very elementary, and it has been realized from the outset that this model is only an approximation to the true situation in W-R stars. Line profile variability, or the production of X-rays, cannot, for example, be addressed with the Standard Model. The model will stand or fall, or undergo modification based on its agreement with observation. Below we summarize the successes and failures of the Standard Model and discuss their implications for our understanding of $\mathrm{W}-\mathrm{R}$ atmospheres.

\subsection{Successes of Standard Model}

(i) The Standard Model provides a good match to the observed continuum of W-R stars (from $6 \mathrm{~cm}$ to $1500 \AA$ ), to the strengths of the He I and He II lines, and to their general profile shapes. The ionization stratification predicted in the models is consistent with observation, and in conjunction with optical depth effects provides an explanation for the different profiles illustrated by different ionization species.

(ii) The ionization structure for metals is in rough agreement with observation. The discrepancies for individual lines are generally at the factor of 2 level (a few lines differ by up to a factor of 5) (Hillier 1988,1989; Wessolowski, Hamann, and Schmutz - these proceedings). In the WC model of Hillier (1989) the C II, C III, $\mathrm{C} \mathrm{IV}, \mathrm{He}$ I and He II spectra were generally reproduced to better than a factor of 2.

\subsection{Problems With Standard Model}

(i) Incorrect metal line strengths: In the WC model of Hillier (1989), several lines of carbon were not produced. For example, the strength of C IV $\lambda 5805$ was underestimated by a factor of 5 . The reason for this discrepancy is unknown. Likewise, some C III line strengths did not agree with observation. For some C III lines (e.g., C III $\lambda 6740)$ this is due to inadequate atomic data. New atomic data from the opacity project, and specifically new C III atomic data computed by Peter Storey (private communication) should help rectify this problem.

Likewise, in WN models it is impossible at the present time to fit all $\mathrm{N}$ lines simultaneously (Hamann, Wessolowski, and Schmutz - these proceedings).

At present there are three basic deficiencies of calculations with the Standard Model. We neglect line blanketing, and line overlap, and accurate atomic data are only now becoming available. As a consequence it is difficult to decide whether these discrepancies provide useful insights into the structure of W-R winds - more detailed and advanced calculations are required.

(ii) P Cygni absorption on the He I and He II lines: For the strong lined WN stars, the models tend to produce a flat topped He I $\lambda 5876$ line profile (as observed), but with a P Cygni absorption much deeper than observed. This is significant, and may indicate a real problem with the Standard Model. The P Cygni profile arises from the large column density along the line of sight to the stellar core - the velocity gradient along this line of sight is small. At present two possible solutions 
present themselves: we need to decrease the column density, or alternatively, allow for a fluctuating velocity field (macro-turbulence). Evidence for fluctuations in the velocity field come from UV lines - the P Cygni absorption is seen to vary in some stars (e.g., St-Louis et al. 1989). As neutral helium is always an impurity species, the presence of density inhomogeneities is unlikely to solve this problem.

A similar problem applies to the He II lines, principally He II $\lambda 1640$. The Standard Model yields a black absorption on the blue side - the observed P Cygni profiles are broader, and are generally not black.

(iii) P Cygni absorption on C III ג1909: As for He I, and He II, the Standard Model produces a deep absorption of the C III $\lambda 1909$ intercombination line which is much stronger than observed (Hillier 1989). Absorption is seen but it is weaker. This may be related to the problem with the P Cygni absorption dip on the He I and $\mathrm{He}$ II lines, but could also be a problem with the ionization structure. In the WC model of Hillier (1989) $\mathrm{C}^{2+}$ becomes the dominant ionization state of carbon in the outer regions of the stellar wind and thus density inhomogeneities might help solve this problem (as column density is less).

(iv) Electron scattering wings: The strength of the electron scattering wings provides a consistency check on the mass loss rates derived from line profile fitting. If the mass loss rates are higher than $10^{-5} M_{\odot} \mathrm{yr}^{-1}$ these wings must exist (Auer and Van Blerkom 1972, Hillier 1984). Because of the expanding atmosphere, and because the scattering is coherent in the frame of the electron, we preferentially see a red wing on W-R emission lines. In $\mathrm{P}$ Cygni stars, which have considerably lower terminal velocities, the electron scattering profile is much more symmetric (see Hillier, McGregor, and Hyland 1988 for an example).

In WN stars two lines provide the best evidence for the wings - He II $\lambda 1640$, and He II $\lambda 5411$. Alternate explanations for the wings have been provided but at the present time these are unsatisfactory. Suggestions that the wing on the He II line at $\lambda 5411$ line is due to OV, C IV, N III, or O III are not satisfactory as lines expected to be stronger are not seen (e.g., Bappu 1973). A peculiar excitation mechanism may operate, but none has been offered.

The electron scattering wings produced in the models of Hillier $(1987 \mathrm{~b}, 1988$, 1989 ) tend to be stronger than observed - possibly by a factor of 2 . If the wing on $\lambda 5411$ is a blend, the discrepancy is even larger. Such discrepancies may be due to density inhomogeneities in the stellar wind - the emission processes scale as the density squared whereas the electron scattering optical depth scales as the density. Electron scattering wings of reduced strength can be obtained in this way (Hillier 1990b).

\section{ABUNDANCES}

From the studies of Willis and Wilson (1978), Nugis $(1975,1982 a, b)$, Smith and Willis $(1982,1983)$, and others, and more recent work by Torres (1988), Smith and Hummer (1988), de Freitas Pacheco and Machado (1988), and Hillier (1988,1989) there can be little doubt that the WN and WC sequences have different elemental abundances. As the status of abundances in W-R stars has recently been critically discussed by Hillier (1990a) I will review the situation only briefly. 
WN stars have enhanced $\mathrm{N}$ and $\mathrm{He}$ (relative to solar abundances), and are deficient in $\mathrm{O}, \mathrm{C}$, and $\mathrm{H}$. Extensive mass loss has revealed, at the surface, matter processed by the CNO nuclear burning cycle. For WNE stars Smith and Willis (1982) found $\mathrm{C} / \mathrm{N}$ ratios of 0.01 to 0.09 by number. For HD 50896, Hillier found from detailed modeling a $\mathrm{C} / \mathrm{N}$ ratio of 0.07 (within a factor of 3 ). These values are in excellent agreement with evolutionary models which give a range from 0.02 to 0.05 (e.g., Maeder 1983, 1987, Prantzos et al. 1986). The $\mathrm{N} / \mathrm{He}$ ratio is more difficult to determine because of the different sensitivity of the $\mathrm{N}$ and He lines to the wind electron temperature. More detailed calculations are required.

WC stars have enhanced $\mathrm{C}, \mathrm{O}$, and $\mathrm{He}$, and are deficient in $\mathrm{H}$, and probably $\mathrm{N}$. In this case, the products of He nuclear burning have been revealed at the surface. $\mathrm{C} / \mathrm{He}$ ratios of $>0.1$ are now favored (Nugis $1982 \mathrm{~b}$, Torres 1988, Smith and Hummer 1988, Hillier 1989). Earlier determinations by Smith and Willis $(1982,1983)$ that the $\mathrm{C} / \mathrm{He}$ ratio was 0.01 to 0.04 underestimate the $\mathrm{C} / \mathrm{He}$ abundance. Smith and Willis used the strength of C III $\lambda 2296$ to constrain the electron temperature but this line is now known to be significantly influenced by dielectronic recombination (Storey 1981, Hillier 1989). Smith and Willis overestimated the wind electron temperature, and hence underestimated the $\mathrm{C} / \mathrm{He}$ abundance. In the WC model of Hillier (1989) there is no disagreement between the strong UV collisionally excited lines, and the optical/IR recombination lines.

The above abundance determinations are entirely consistent with the observed properties of W-R stars. At least four observational arguments lend strong support to the interpretation that $\mathrm{W}-\mathrm{R}$ stars are evolved objects in which mass loss has revealed nuclear processed material at the surface, that many W-R stars (but not all) contain no $\mathrm{H}$, and that the $\mathrm{WN}$ and $\mathrm{WC}$ sequences are a direct result of different surface compositions.

Massey and Conti (1980) found that the WN8 star HD177230 (which shows emission due to N II, N III, N IV, N V and He I) is severely hydrogen deficient. This star has an excitation similar to other WN8 stars that clearly show the presence of hydrogen. The only non-contrived means of explaining this observation is on the basis of different $\mathrm{H}$ abundances.

Bhatia and Underhill $(1986,1988)$ argue that the difference in strengths of $\mathrm{N}$ emission lines in WN and WC stars is primarily due to different wind electron temperatures, and is not an abundance indicator. This is contradicted by observation. In HD 165763 emission due to O VI $\lambda 3811,3834$ is present (Bappu 1973) but in HD 50896 (WN5) such emission is not detectable. If HD 50896 has a higher excitation than HD 165763 (as argued by Bhatia and Underhill on the basis of the strong $\mathrm{N} \mathrm{IV,} \mathrm{N} \mathrm{V} \mathrm{emission} \mathrm{line} \mathrm{spectrum),} \mathrm{why} \mathrm{are} \mathrm{the} \mathrm{higher} \mathrm{excitation} \mathrm{O}$ VI emission lines not seen (Hillier 1990)?

The WC5 star HD 165763 (WC5) and the WN5 star HD 50896 are characterized by very different $\mathrm{C}$ and $\mathrm{N}$ spectra (as expected by their spectral types). However, the $\mathrm{Fe} \mathrm{V}$ and $\mathrm{Fe}$ VI emission spectrum at $\mathrm{UV}$ wavelengths (Fig. 1) is very similar. As $\mathrm{Fe} \mathrm{V}$ has an ionization potential very similar to that of $\mathrm{N}$ IV, while that of Fe VI is virtually identical to $\mathrm{N} \mathrm{V}$, it is difficult to see how excitation effects can be invoked to explain the observed spectral differences between these two stars. The importance of Fe IV, Fe V, and Fe VI in W-R stars has previously been emphasized by Nugis and Sapar (1985a,b), Eaton, Cherepaschuk, and Khaliullin (1985a,b) and 


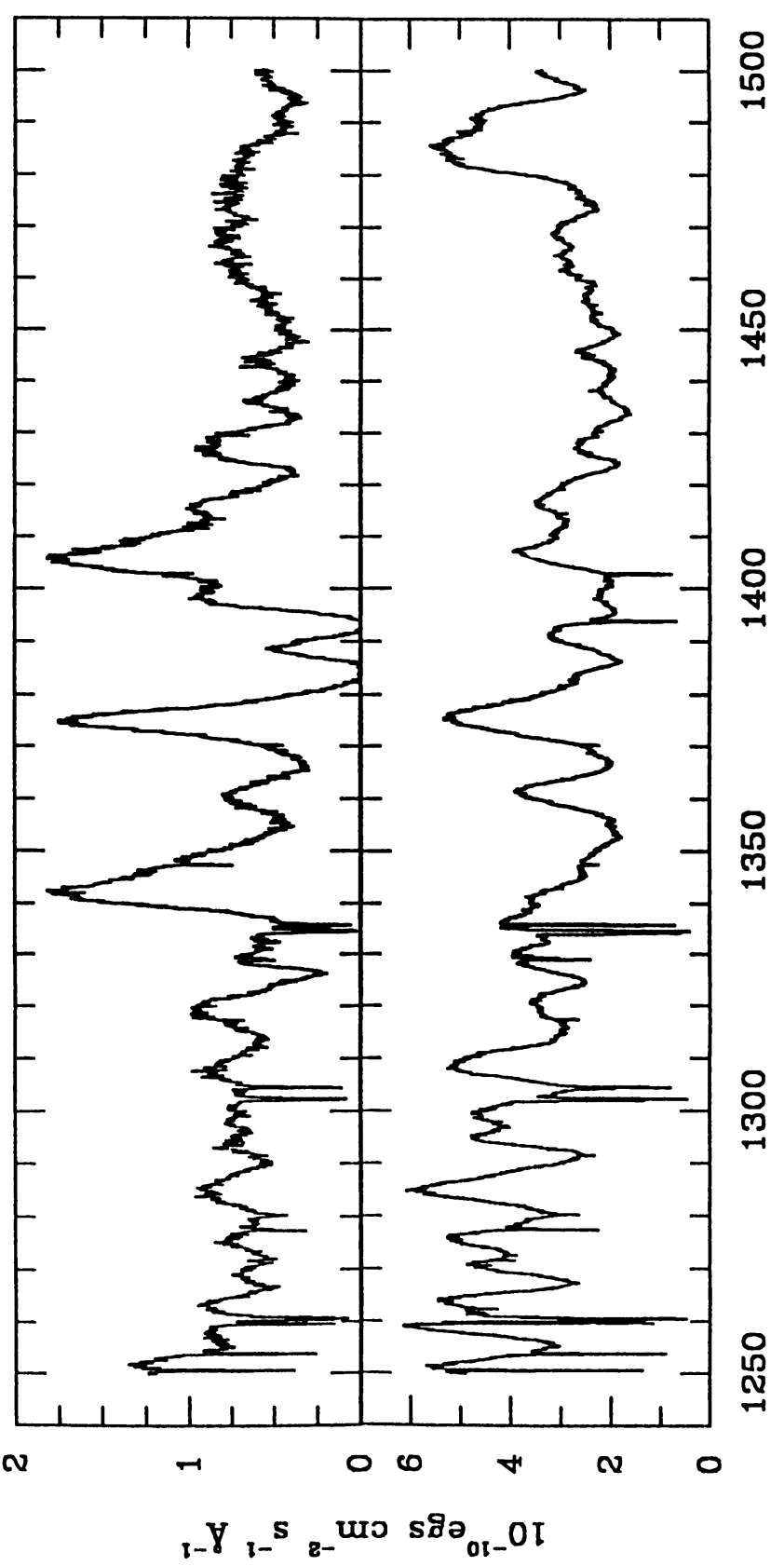

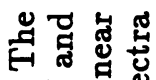

$\dot{0}$ 뜽요

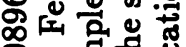

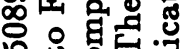

ช 8

因艺藻

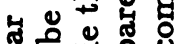

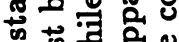

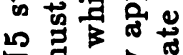

之 100 ?

3 \&

ฮ

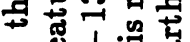

पथ

대용

晋

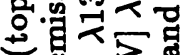

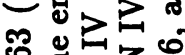

๕

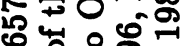

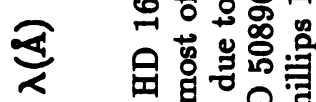

굴

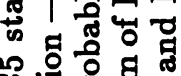

ว 2.0

3 造击

ข 局造

혼 응

4

०

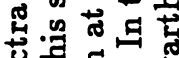

영

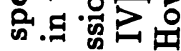

뫃

릉.ตำ

的娄

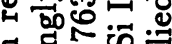

드를

부분 웅

的团

모모․

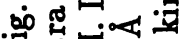

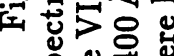
언 
Koenigsberger and Auer (1985).

Finally, we note that $\mathrm{N}$ lines are seen in the spectra of some WC and WO central stars of planetary nebulae (Heap 1982, Barlow and Hummer 1982). This suggests that the WC sequence is not a result of excitation - rather the absence of $\mathrm{N}$ in population I WC spectra indicates a low N/C abundance ratio.

\section{ACCURACY OF STELLAR PARAMETERS}

The accuracy of current stellar parameter determinations is very difficult to ascertain. There are several reasons for this:

(i) The bulk of the flux in the early W-R stars is unobservable, and hence model calculations must be used to constrain the luminosity and effective temperature.

(ii) The mass loss mechanism is still not understood. What drives the wind, and why can the observed wind momenta exceed the single scattering limit by a factor of 40 ?

(iii) It is difficult to determine the significance of discrepancies between theory and observation.

(iv) The models to date neglect line blanketing which must play an important role in determining the atmospheric structure.

Firstly, model mass loss rates are comparable to radio mass loss to better than $50 \%$, although it appears that the model mass loss rates may be upper estimates. The principal uncertainty with the mass loss rates is the importance of clumping - hopefully analysis of electron scattering wings and other density sensitive diagnostics will allow strong constraints to be placed on the importance of this effect.

With regard to bolometric corrections, Schmutz (1990) argues that they are incorrect by at most 1 magnitude. He bases his conclusion on a comparison of different models made with different assumptions, and on the basis of preliminary line blanketing calculations. Bolometric magnitudes and effective temperatures are likely to be more accurate for the cooler $\mathrm{W}-\mathrm{R}$ stars, and those with relatively weak (i.e., low density) winds (e.g., WN 7 stars).

Support for the luminosities and effective temperatures derived from the Standard Model comes from analyses of nebulae. Smith and Clegg (1990), Rosa and Mathis (1990), and Vogel (1990) find $T_{\text {eff }}$ 's compatible with that found by the Kiel group.

Of fundamental concern for the radii and effective temperatures we derive is the difficulty in relating these parameters to those adopted in evolutionary calculations. Until we understand the wind dynamics this cannot be achieved. It is the strong lined WN stars, and the WC stars that are most affected by the atmospheric extension.

This problem is highlighted by some recent calculations made by Hillier to address observational and theoretical constraints on the velocity law in W-R stars. A model with $L=10^{5} L_{\odot}, R_{*}=2.5 R_{\odot}$ (i.e., $T_{\text {eff }}=65,000 \mathrm{~K}$ ), $\dot{M}=5 \times$ $10^{-5} M_{\odot} \mathrm{yr}^{-1}, V_{\infty}=1700 \mathrm{~km} \mathrm{~s}^{-1}$, and $\beta=1$ (exponent in standard velocity law) was found to have a continuum, and line profiles (for selected lines of He I, He II, C IV, N IV, and N V) observationally indistinguishable (see Fig. 2) from a model of the same luminosity and mass loss rate, but with $R_{*}=1 R_{\odot}$ 
(i.e., $\left.T_{\text {eff }}=100,000 \mathrm{~K}\right), V_{\infty}=1800 \mathrm{~km} \mathrm{~s}^{-1}$, and a slow velocity law characterized by $\beta=3$. Clearly, we need a dynamic model to help constrain the velocity law, and to allow reliable determinations of effective temperatures for comparison with evolutionary calculations.
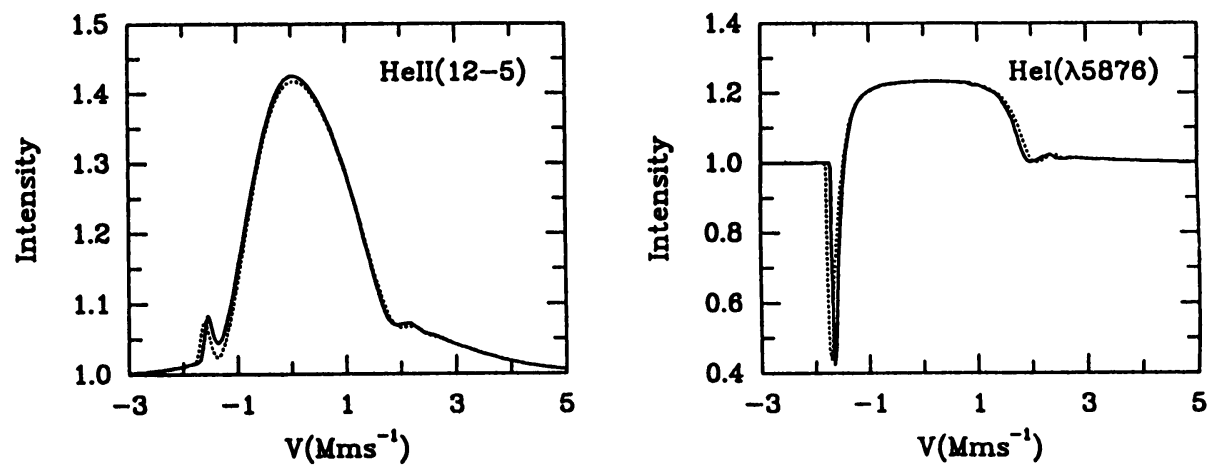

Fig. 2.- Example line profiles computed for two models characterized by different velocity laws and core radii. The solid line has $R_{*}=2.5 R_{\odot}, V_{\infty}=$ $1700 \mathrm{~km} \mathrm{~s}^{-1}$, and $\beta=1$, while the broken line is for a model with $R_{*}=1.0 R_{\odot}$, $V_{\infty}=1800 \mathrm{~km} \mathrm{~s}^{-1}$, and $\beta=3$. The profiles are observationally indistinguishable. Similar comments apply to other lines such as N V $\lambda 1240$, N V $\lambda 4609$, N IV $\lambda 1486$, and C IV $\lambda 1549$.

\section{CONCLUSION}

Since the last IAU Symposium on W-R stars our knowledge and understanding of these stars has dramatically increased. Sophisticated models can now be used to provide essential diagnostic information regarding $W-R$ stellar parameters, and the structure of W-R stellar winds. Improvements to the codes are in progress, and it is hoped that these, combined with high quality observations over a wide spectral range, will allow further significant advances.

The Standard Model used to represent the extended atmosphere of a W-R star is an excellent first approximation. The ionization stratification, and the importance of ionizations from excited states will also appear in more complicated models. Inferences concerning the structure of W-R stellar atmospheres which are based on models that do not allow for these effects must be treated with caution. 


\section{ACKNOWLEDGEMENTS}

The author wishes to thank Drs. Peter Storey, David Hummer, and Werner Schmutz for invaluable discussions. Thanks to Lorraine Volsky for editorial assistance. This work was supported by National Science Foundation grant AST8802937 through the University of Colorado. The computations were done on the JILA VAX 6440.

\section{REFERENCES}

Abbott, D. C., and Hummer, D. G. 1985, Ap. J., 294, 286

Antokhin, I. I., Kholtygin, A. F, and Cherepaschuk, A. M. 1988, Astr. Zh., 65, 558 (Sov. Astr., 32, 285)

Auer, L. H., and Van Blerkom, D. 1972 Ap. J., 178, 175

Bappu, M. K. V. 1973, in IAU Symposium 49, Wolf-Rayet and High Temperature Stars, ed. M. K. Bappu and J. Sahade (Boston: Reidel), p. 59

Barlow, M. J., and Hummer, D. G. 1982, in IAU Symposium 99, Wolf-Rayet Stars: Observations, Physics, Evolution, ed. C. W. H. de Loore, and A. J. Willis (Dordrecht: Reidel), p. 387

Beals, C. S. 1944, M.N.R.A.S., 104, 205

Bhatia, A. K., and Underhill, A. B. 1986, Ap. J. Suppl., 60, 323

Bhatia, A. K., and Underhill, A. B. 1988, Ap. J. Suppl., 67, 187

Eaton, J. A., Cherepaschuk, A. M., and Khaliullin, Kh. F. 1985a, Ap. J., 296, 222

Eaton, J. A., Cherepaschuk, A. M., and Khaliullin, Kh. F. 1985b, Ap. J., 297, 266(erratum 1988, 334, 1076)

de Freitas Pacheco, J. A., and Machado, M. A. 1988, A. J., 96, 365

Hamann, W.-R., 1985, Astr. Ap., 145, 443

Hamann, W.-R., and Schmutz, W. 1987, Astr. Ap., 174, 173

Hamann, W.-R., Schmutz, W., and Wessolowski U. 1988, Astr. Ap., 194, 190

Hamann, W.-R., and Wessolowski, U. 1990 Astr. Ap., 227, 171

Heap, S. R. 1982, in IAU Symposium 99, Wolf-Rayet Stars: Observations, Physics, Evolution, ed. C. W. H. de Loore, and A. J. Willis (Dordrecht: Reidel), p. 423

Hillier, D. J. 1983, Ph. D. thesis, Australian National University, Canberra

Hillier, D. J. 1984, Ap. J., 280, 744

Hillier, D. J. 1987a, Ap. J. Suppl., 63, 947

Hillier, D. J. 1987b, Ap. J. Suppl., 63, 965

Hillier, D. J. 1988, Ap. J., 327, 822

Hillier, D. J. 1989, Ap. J., 347, 392

Hillier, D. J. 1990a, in Properties of Hot Luminous Stars, ed. C. D. Garmany (Astron. Soc. Pacific Conf. Series, Vol. 7), p. 340

Hillier, D. J. 1990b, in preparation

Hillier, D. J., Jones, T. J., and Hyland, A. R. 1983 Ap. J., 271, 221

Hillier, D. J., McGregor, P. J., and Hyland, A. R. 1988, Mass Outflows from Stars and Galactic Nuclei, ed. L. Bianchi and R. Gilmozzi, (Dordrecht: Kluger), p. 215 
Hogg, D. E. 1985, in Radio Stars, ed. R. M. Hjellming and D. M. Gibson (Dordrecht: Reidel), p. 117

Hogg, D. E. 1989, A. J., 98, 282

Howarth, I. D., and Phillips, A. P. 1986, M.N.R.A.S., 222, 809

Koenigsberger, G., and Auer, L. H. 1985, Ap. J., 297, 255

Kuhi, L. V. 1968, in Wolf-Rayet Stars, ed. K. B. Gebbie and R. N. Thomas (NBS SP-307), p. 101

Kuhi, L. V. 1973, in IAU Symposium 49, Wolf-Rayet and High Temperature Stars, ed. M. K. Bappu and J. Sahade (Boston: Reidel), p. 205

Maeder, A. 1983, Astr. Ap., 120, 113

Maeder, A. 1987, Astr. Ap., 173, 247

Massey, P., and Conti, P. S. 1980, Ap. J., 242, 638

Nugis, T., 1975, in IAU Symposium 67, Variable Stars and Stellar Evolution, ed. V. E. Sherwood and L. Plaut (Dordrecht: Reidel), p. 291

Nugis, T., 1982a, in IAU Symposium 99, Wolf-Rayet Stars: Observations, Physics, Evolution, ed. C. W. H. de Loore and A. J. Willis (Dordrecht: Reidel), p. 127

Nugis, T., 1982b, IAU Symposium 99, Wolf-Rayet Stars: Observations, Physics, Evolution, ed. C. W. H. de Loore, and A. J. Willis (Dordrecht: Reidel), p. 131

Nugis, T., and Sapar, A. 1985a, Pis'ma Astron. Zh. 11, 455 (Sov. Astron. Lett. 11, 188)

Nugis, T., and Sapar, A. 1985b, Tartu Astrofüüs. Obs. Teated Nr. 80

Prantzos, N., Doom, C., Arnould, M., and de Loore, C. 1986, Ap. J., 304, 695

Rosa, M. R., and Mathis, J. S. 1990, in Properties of Hot Luminous Stars, ed. C. D. Garmany (Astron. Soc. Pacific Conf. Series, Vol. 7), p. 135

Rublev, S. V., 1964, Astr. Zh., 41, 63(Sov. Astr., 8, 45)

Schmutz, W. 1990, in Properties of Hot Luminous Stars, ed. C. D. Garmany (Astron. Soc. Pacific Conf. Series, Vol. 7), p. 117

Schmutz, W., and Hamann, W.-R. 1986, Astr. Ap., 166, L11

Schmutz, W., Hamann, W.-R., and Wessolowski, U. 1989, Astr. Ap., 210, 236

Smith, L. F, and Clegg, R. E. S. 1990, in Properties of Hot Luminous Stars, ed. C. D. Garmany (Astron. Soc. Pacific Conf. Series, Vol. 7), p. 132

Smith, L. F., and Hummer D. G. 1988, M.N.R.A.S., 230, 511

Smith, L. J., and Willis A. J. 1982, M.N.R.A.S., 201, 451

Smith, L. J., and Willis A. J. 1983, Astr. Ap. Suppl., 54, 229

St-Louis, N., Smith, L. J., Stevens, I. R., Willis, A. J., Garmany, C. D., and Conti, P. S. 1989, Astr. Ap., 226, 249

Storey, P. J. 1981, M.N.R.A.S., 195, 27P

Torres, A. V. 1988, Ap. J., 325, 759

Vogel, W. 1990, in Properties of Hot Luminous Stars, ed. C. D. Garmany (Astron.

Soc. Pacific Conf. Series, Vol. 7), p. 129

Wessolowski, U., Schmutz, W., and Hamann, W.-R. 1988, Astr. Ap., 194, 160

Williams, P. M., van der Hucht, K. A., Sandell, G., and Thé, P.S. 1990, M.N.R.A.S., 244, 101

Willis, A. J., and Wilson, R. 1978, M.N.R.A.S., 182, 559 


\section{DISCUSSION}

Pollock: With regard to abundance measurements, I would encourage you to have a look at the X-ray measurements of HD 193793 of Williams et al. (1990, MNRAS, 243, 662) where an X-ray source suffers increased absorption as it moves deeper into the wind of the WC7 star. This gives an essentially ionization independent abundance measurement of $N_{c} \sim 0.06$.

Hillier: It would be of use to compare the above abundance with that derived from a detailed spectroscopic analysis. While the abundance you derive is ionization independent, it will contain other model dependencies.

Underhill: You remarked that the large discrepancies with the absorption components predicted by you are "no serious limitation"! I disagree entirely. They are a significant test of your model's physical state; they show that the models fail. The fact that $\dot{M} v_{\infty} c / L$ is of the order of 40-50 in many of the Kiel models and your models is a disaster. You imply that there is a strong force, unknown to physics, present to drive the wind. I refuse to believe in presently unknown long-range forces of physics.

Hillier: We are well aware of the discrepancies of the model with observation, however the "Standard Model" describes the spectrum too well for it to be seriously in error. One purpose of the "Standard Model" is to identify such discrepancies so that we can further our understanding of WR stellar winds. The problem with the absorptions is principally one of degree - strong P Cygni absorption is seen in the HeI $\lambda 5876$ line in HD 192163. The observed variability of the P Cygni profiles, both in the UV and optical, clearly show that the intrinsic instability of radiation winds is having a significant influence on the line profiles. We are currently addressing the implications for our analyses. As regards the momentum problem, it must be emphasized that the single scattering limit is not an absolute limit to the momentum that can be delivered to the stellar wind (see Cassinelli, this symposium). In WR stars multiple scattering will be very important in determining the wind dynamics. We also need to consider line overlap, the diffuse radiation field and the large optical depth of the stellar wind at the sonic point. Whether these effects can lead to the large momentum depositions required is still an open question. We also need to consider the errors in the determinations of $\dot{M}$ and $L$. It is possible that the single scattering limit is exceeded by only a factor of 10 , rather than the factor of 40 currently found for some objects.

Langer: Many WR stars have wind densities as high that the optical depth (continuum) close to the stellar surface must be high and you cannot observationally determine the velocity law in this part of the wind. How does this uncertainty affect your results, especially results on $R_{*}$ ?

Hillier: This question was already answered in my talk. 


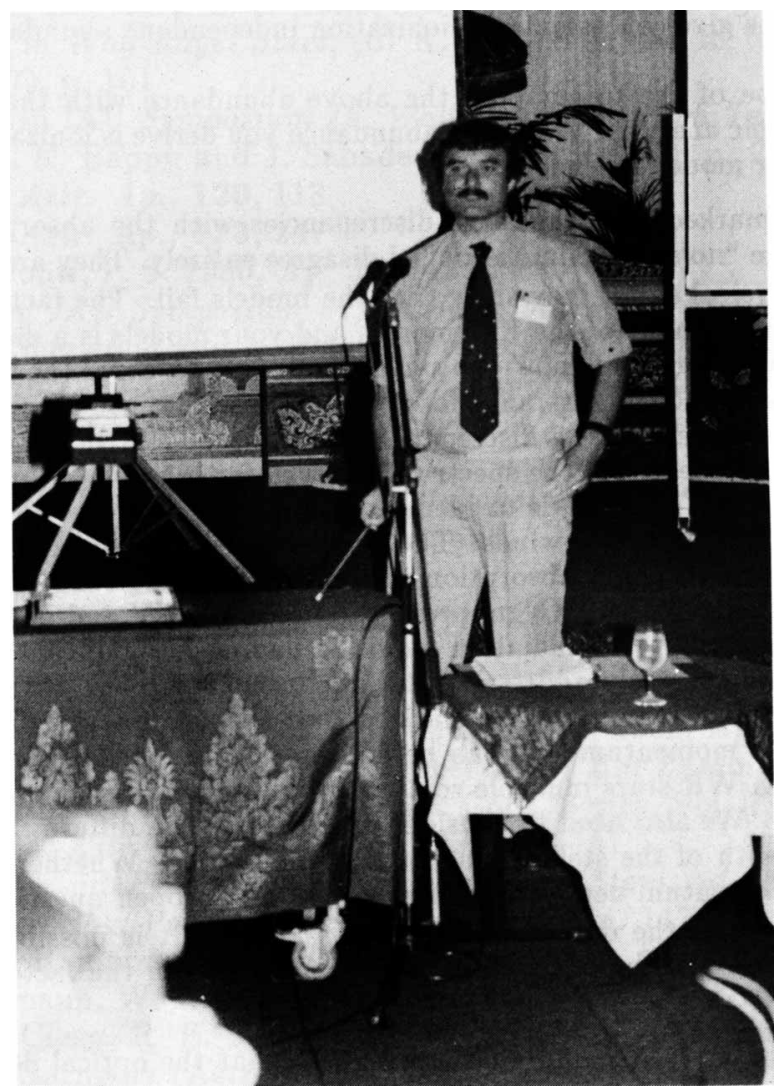

John Hillier 